\title{
Study of the proton reconstruction efficiency with the ALFA detector using an overlay technique of Monte Carlo signal events with zero-bias collider data
}

Inga Łakomiec*, on behalf of the ATLAS Collaboration

AGH University of Science and Technology, Kraków, Poland

E-mail: inga.katarzyna.goralczyk@cern.ch

The purpose of the ALFA detector is to measure protons scattered at very small angles. ALFA aims to study elastic and diffractive events, exclusive production and photon induced interactions. In LHC Run 2, this detector participated in the ATLAS high-luminosity data taking. In addition, several special runs with reduced luminosity were taken. Any cross section measurement requires good understanding of the particle reconstruction efficiency. This task is particularly complicated in the case of forward protons where the actual beam condition is an important part of the working environment. The modelling of the very forward region in terms of primary particle flux and inactive material producing secondary particle is generally not precise. An overlay technique of Monte Carlo signal events with zero-bias collider data has overcome these difficulties and may provide better understanding of the proton reconstruction efficiency. This report presents results of the proton reconstruction efficiency with the ALFA detector during LHC Run 2.

7th Annual Conference on Large Hadron Collider Physics - LHCP2019

20-25 May, 2019

Puebla, Mexico

${ }^{*}$ Speaker. 


\section{Introduction}

Particle reconstruction efficiency calculations play a crucial role in any cross section measurement. They pose some difficulties especially in the forward region where the actual beam conditions are an important part of the working environment. This task has become more complicated with higher luminosity and the associated higher pile-up. The average number of inelastic $p p$ interactions per bunch crossing $\mu$ was up to 70 in Run 2 [1], and it will increase further in Run 3. Values of $\mu$ can approach up to 200, during the High-Luminosity LHC operations [2].

The ALFA detector, whose layout is presented in Figure 1, is one of the ATLAS Roman Pot detectors [3]. It is designed to measure protons scattered at very small angles. Elastic and diffractive events, exclusive production and photon induced interactions can be studied using the detectors placed on both sides of ATLAS about $240 \mathrm{~m}$ from the ATLAS Interaction Point [4].

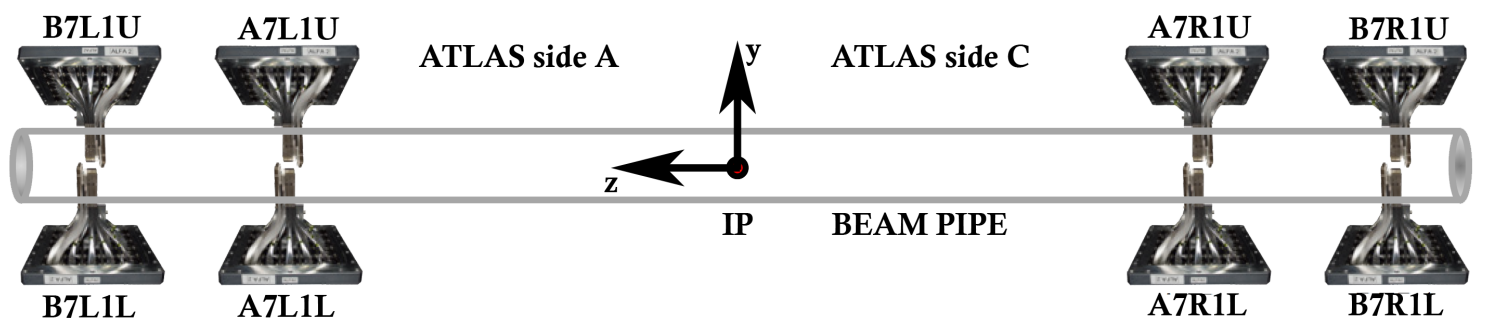

Figure 1: Naming convention of the ALFA detectors. Letter A indicates inner, B - outer, U - upper and L lower ALFA stations [5].

So far pileup has been simulated based on minimum-bias Monte Carlo (MC). In general, this procedure might not be precise. There are several reasons why the minimum-bias MC simulation like PYTHIA is inadequate in the forward direction. Firstly, the primary forward particle flux is not precisely known. Secondly, a description of the inactive material implemented in the simulation is only an approximation of a complex beamline system.

The overlay technique used in this study is a mix (on a hit level) of MC signal events and zero-bias collider data taken in parallel with the physics trigger on the timing of bunch crossing. The scheme of the process is shown in Figure 2.

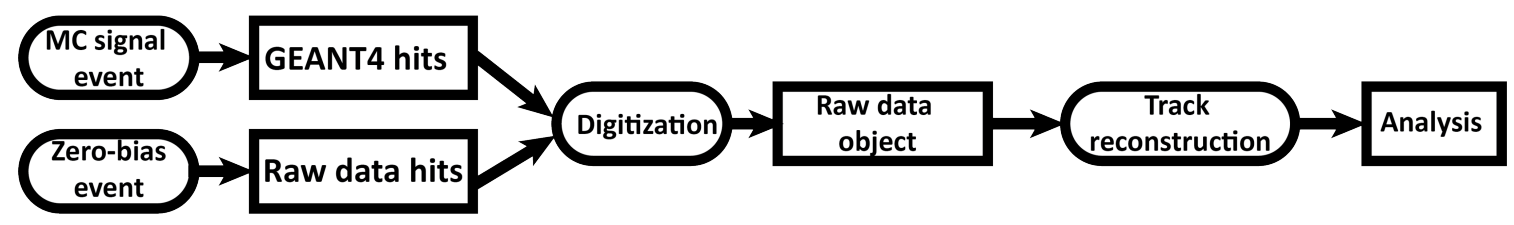

Figure 2: A scheme of combining zero-bias collider data with MC signal events.

Such procedure is an alternative method for pileup simulation and may provide better understanding of proton reconstruction efficiency and migration effects due to actual experimental conditions. 


\section{Proton reconstruction in ALFA}

Each of eight ALFA detectors consists of ten scintillating fibre modules with two layers of 64 orthogonally arranged fibres designed to measure transverse positions of the forward protons. A hit pattern of a proton trajectory in the layers of one fibre orientation is shown in Figure 3.

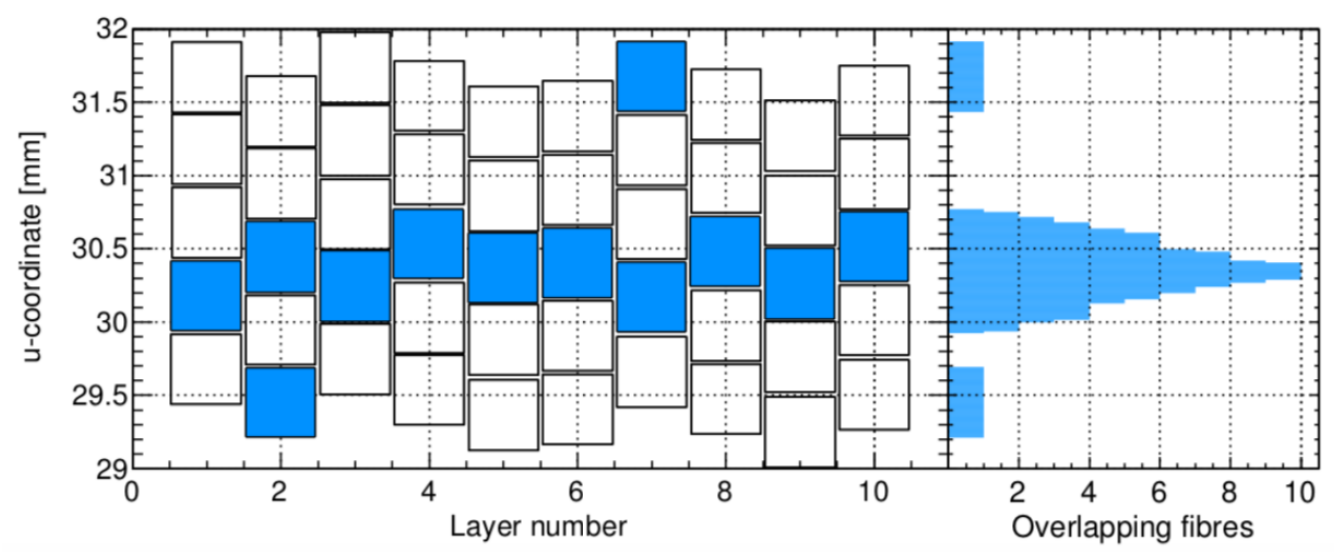

Figure 3: A hit pattern of a proton trajectory in ten layers of one fibre orientation. The superposition of active fibres attributed to a track is shown in the histogram. The position of maximum overlap is used to determine the track position [6].

It is possible to apply different track reconstruction methods to optimise the analysis efficiency. Two such methods of a track selection have been examined in this study. The first one is a multitrack selection where the longest track with at least 4 associated active fibres in each orientation in the given station is taken as a proton candidate, the second one is a single-track selection where the track is required to have at least 5 associated active fibres in each orientation and no additional such tracks are present in the given station. An advantage of the multi-track selection method is higher efficiency compared to the single-track selection. On the other hand, this method has lower purity.

\section{Overlay in ALFA}

The validation of the overlay procedure has been performed using data collected in October 2015 during the LHC fill 3846 and the special run with following parameters: $\beta^{*}=90 \mathrm{~m}, \mu=0.08$, the distance of the inner ALFA station from the beam $\sim 6.85 \mathrm{~mm}$. Exactly the same conditions, except pileup which was set to $\mu=0$, have been used in simulations. The comparison of the active fibre multiplicity between PYTHIA 8 elastic MC simulation without overlay and MC simulation with overlaid zero-bias collider data is shown in Figure 4. Despite relatively small pileup, there is a shift towards larger values of multiplicities for a distribution obtained with overlay. A clear peak around 40 which came from events with two overlaid protons is also visible on the plot. 


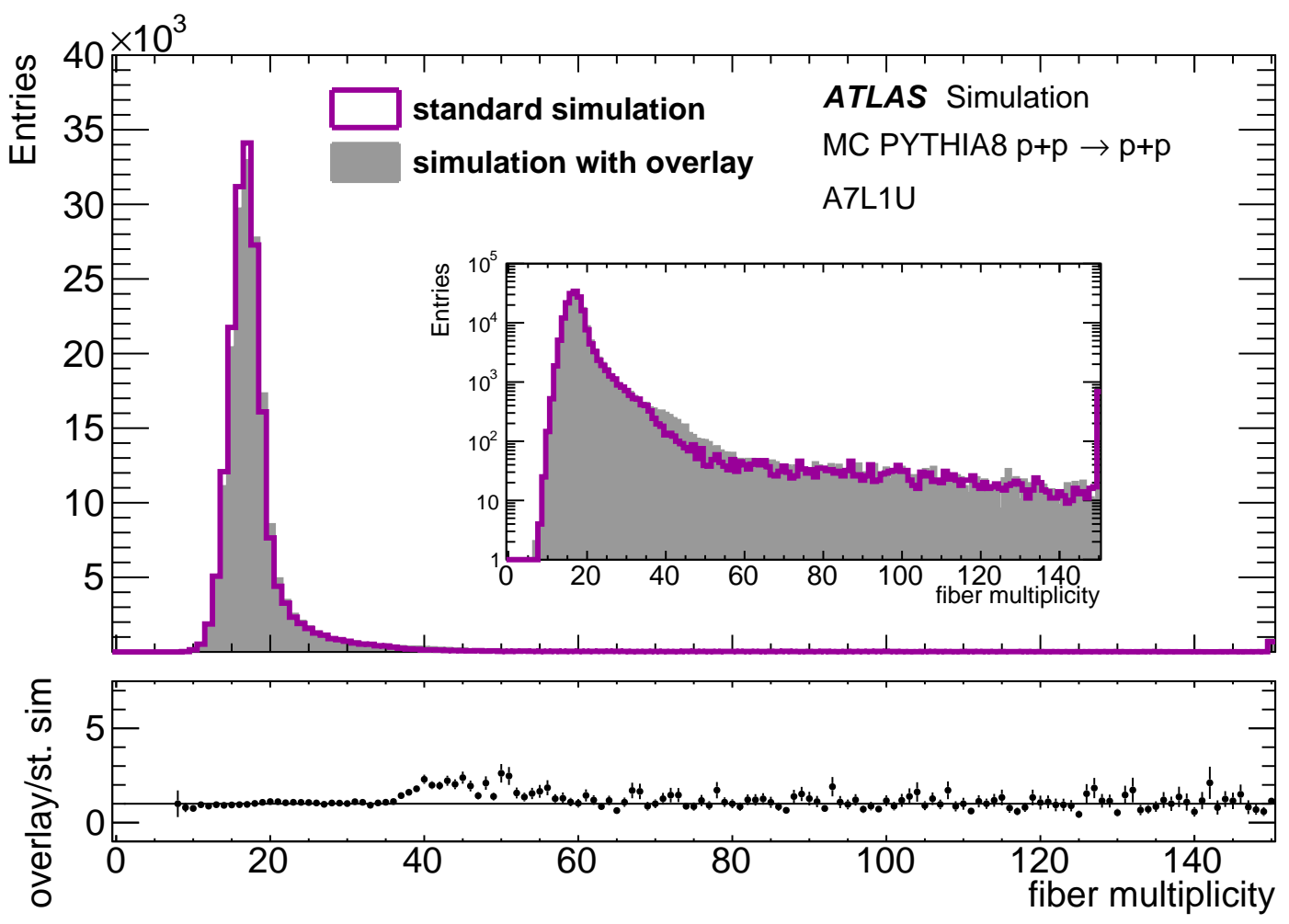

Figure 4: Distribution of active fiber multiplicity for A7L1U inner station for events with at least 5 active fibres. The bottom panel shows the ratio of both distributions.

\section{Proton reconstruction efficiency}

Elastic events have been used to compare simulations with real data. Only events with no signal in the ATLAS Inner Detector and Minimum Bias Trigger Scintillators (MBTS) have been accepted to suppress an inelastic background. The trigger was simulated by requirement of at least 5 fired fibres in both orientations in the ALFA station where a true proton was expected. Then one of two track selection methods described in Section 2 was used. Such elastic events have been selected first by a requirement of the correlation between the proton candidates' hit positions in inner and outer stations (armlet) on the same ATLAS side expected for beam energy protons. Then the proton candidates from opposite ATLAS sides (arm) have been required to show the back-to-back topology anticipated for elastic scattering. A full reconstruction requires a proton reconstruction in each of four stations from the given elastic arm.

Reconstruction inefficiencies in simulation and data have been studied based on events with a missing proton candidate in one or two stations. For such events it is still possible to select elastic events with the subset of elastic event selection cuts which has been employed here [4]. Two cases of a missing track have been investigated: the first one (case 3/4) when no track was reconstructed in one station and the second one (case 2/4) when there were missing tracks in two stations from the same armlet. These numbers of events have been divided by the number of fully reconstructed 
events (case 4/4). The ratios $\mathrm{N}(3 / 4) / \mathrm{N}(4 / 4)$ and $\mathrm{N}(2 / 4) / \mathrm{N}(4 / 4)$ for one of elastic arms are presented in Figure 5. Other cases of a missing track have not been taken into account because they are insignificant in this analysis.
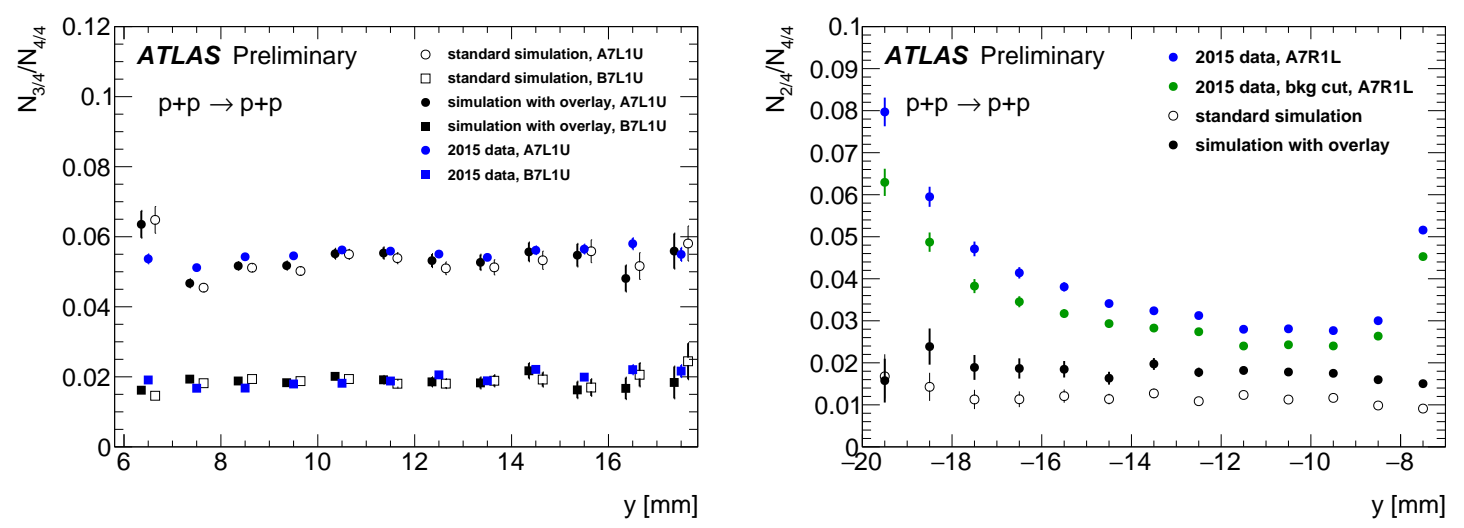

Figure 5: The ratio of a position distribution in $y$ of case 3/4 for A7L1U station where no track was reconstructed in station B7L1U (circles) and for B7L1U station where no track was reconstructed in station A7L1U (squares) to the fully reconstructed elastic case 4/4 in arm B7L1U-A7L1U- A7R1L-B7R1L (the left panel) and the ratio of a position distribution in $y$ of case 2/4 for A7R1L station where no track was reconstructed in armlet B7L1U - A7L1U to the fully reconstructed elastic case 4/4 in arm B7L1U- A7L1UA7R1L-B7R1L (the right panel).

The number of events from case 3/4 makes up about three fourth of the total number of failed elastic events in comparison to case $2 / 4$ where it is one fourth. Showers started in the inner detectors are a frequent reason for a failed reconstruction in the outer stations and that is why ratio $3 / 4$ to $4 / 4$ is higher when the proton is not reconstructed in one of the outer stations. Sometimes showers are induced also before the inner stations and it results in the inability to reconstruct tracks in either one or two detectors. Case 3/4 is well described by MC simulation with and without overlay, in contrast to case $2 / 4$ when the simulation of only elastic process does not describe the data. In the last case a simulation with overlay is closer to data than a simulation without overlay but a significant contribution of an inelastic background can be still present even after applying an additional inelastic background cuts shown as green circles in Figure 5. The inelastic background is expected to be large close to the detector edges. A background-track $y$-position distribution has two components which are showers with very small $y$ and a relatively flat inelastic background with high and medium $y$. An elastic-track $y$-position distribution has high values for small $y$ positions and lower ones for bigger $y$. The background to signal ratio shape comes directly from the $y$ signal and background distributions and has a minimum in the middle of $y$ position distribution and increases when one is closer to edges of the detector. That is why the best estimation of the difference in the reconstruction between data and simulation is visible in the center of the detector. In the central region simulation with overlay describes ratios of case $2 / 4$ to $4 / 4$ observed in real data with precision $\sim 1 \%$.

The reconstruction efficiency (probability that proton(s) expected in a given armlet (arm) was 
(were) reconstructed there) and a fraction of tracks reconstructed $3.5 \sigma(360 \mu \mathrm{m}$ for $x$ and 140 $\mu \mathrm{m}$ for $y$ track positions) away of detector resolution from the expected proton position (wrongly reconstructed tracks) were calculated after validation of the simulation based on elastic events. The results of the study are presented for armlet A7L1U-B7L1U in Figure 6 and for elastic arm B7L1U-A7L1U-A7R1L-B7R1L in Figure 7.
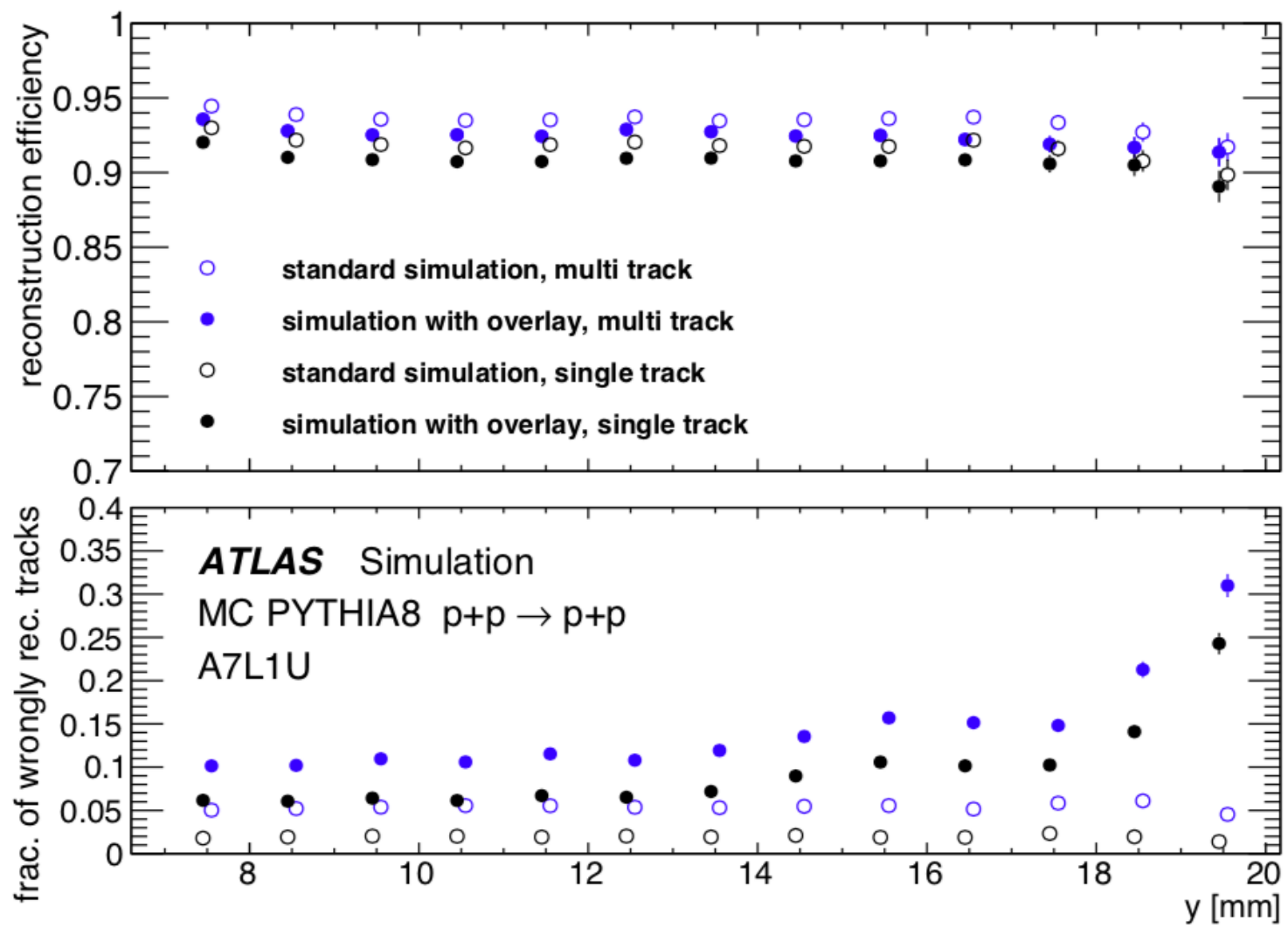

Figure 6: Reconstruction efficiency (top) and fraction of wrongly reconstructed tracks (bottom) for armlet A7L1U-B7L1U.

The proton reconstruction efficiency (Figure 6) predicted by the MC simulation without overlay is $\sim 94 \%$ for a multi-track selection method and $\sim 92 \%$ for a single-track selection method. The elastic event reconstruction efficiency (Figure 7) is lower than the proton reconstruction efficiency because in the latter one requires only one good reconstructed elastic proton instead of both. The MC simulation predicts the elastic event efficiency of $\sim 90 \%$ for a multi-track selection method and $\sim 86 \%$ for a single-track selection method. The efficiency for simulation with overlay is $\sim 2 \%$ lower.

The fraction of wrongly reconstructed tracks for one armlet (Figure 6) predicted by the simulation without overlay is $\sim 5 \%$ for a multi-track selection method and $\sim 2 \%$ for a single-track method. The value is higher for the simulation with overlay by $\sim 5 \%$ rising to $\sim 25 \%$ at the edge of the detector. The fraction concerning the whole elastic arm (Figure 7 ) is $\sim 1.5 \%$ for a multitrack selection method and $\sim 1 \%$ for a single-track method for the simulation without overlay. The simulation with overlay shows $\sim 5 \%$ higher values of the ratio. 

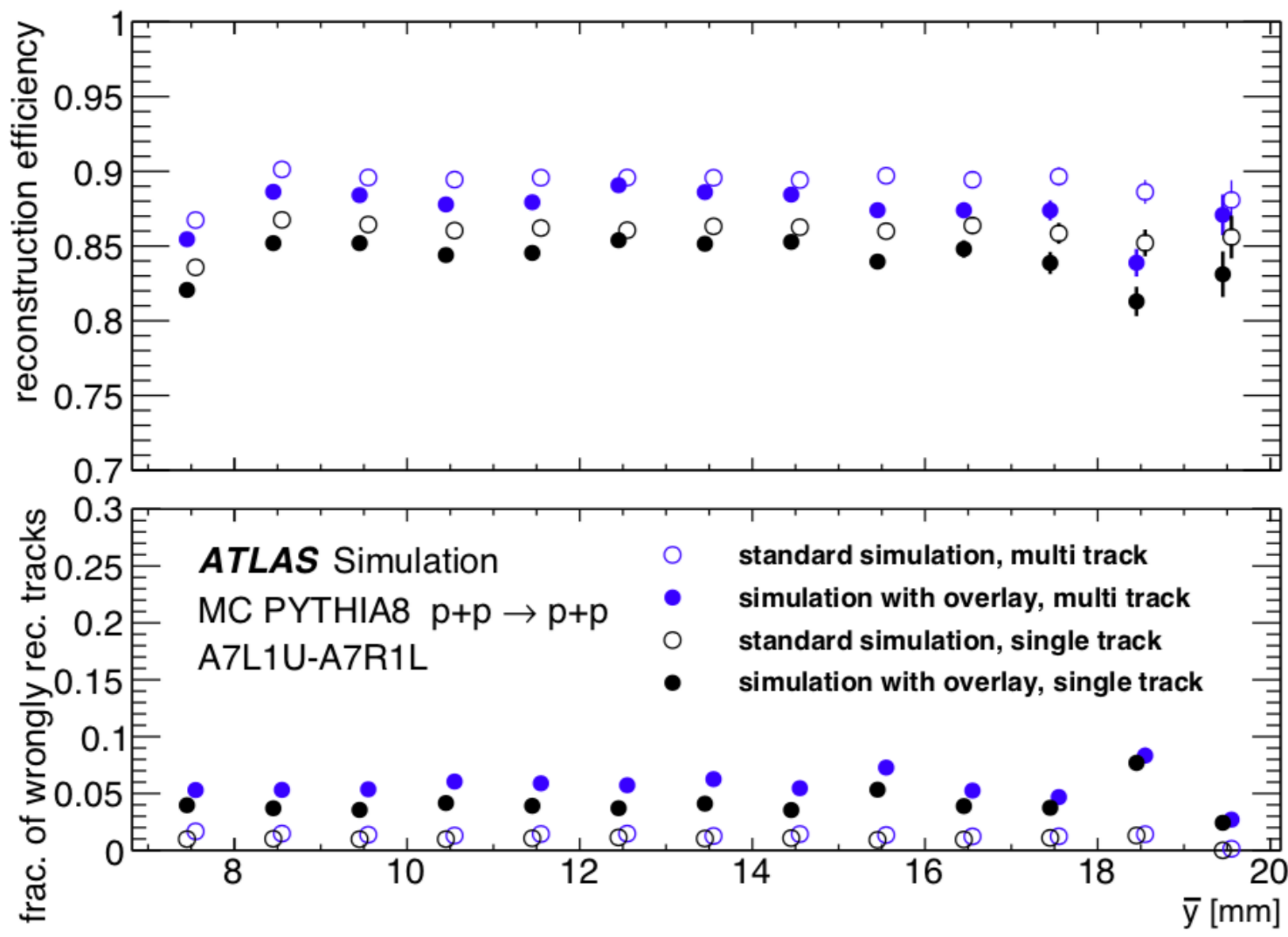

Figure 7: Reconstruction efficiency (top) and fraction of wrongly reconstructed tracks (bottom) for elastic arm B7L1U-A7L1U-A7R1L-B7R1L as a function of average track $y$ positions in A7L1U-A7R1L inner stations.

\section{Conclusions}

The overlay technique has been applied for simulation of the ALFA detector as a part of the ATLAS Roman Pot detectors. The overlay technique improves the precision of simulation of the very forward region compared to a previously used method. It is extremely important especially for higher luminosity expected in further LHC runs. PYTHIA 8 elastic MC simulation without and with overlay zero-bias collider data have been compared in terms of proton reconstruction efficiency and fraction of wrongly reconstructed track calculations. There are visible effects on proton reconstruction efficiency and migration effects due to the actual experimental conditions even at relatively small event pileup of $\mu=0.08$. Overlay is a promising analysis technique for data taken with much larger pileup achieved in LHC Run 2 and expected in Run 3.

\section{Acknowledgments}

This work is supported in part by Polish National Science Center grant no. 2015/19/B/ST2/00989, by a joint project of the Polish National Agency for Academic Exchange and AGH UST no. PPI/PRO/2018/1/00026/U/001, and by PL-Grid infrastructure. 


\section{References}

[1] ATLAS Collaboration, LuminosityPublicResultsRun2, https://twiki.cern.ch/twiki/bin/view/AtlasPublic/LuminosityPublicResultsRun2

[2] ATLAS Collaboration, Expected performance for an upgraded ATLAS detector at High-Luminosity LHC. ATLAS-PUB-2015-026, url: https://cds.cern.ch/record/2055248.

[3] ATLAS Collaboration, The ATLAS Experiment at the CERN Large Hadron Collider, JINST 3 (2008) S08003.

[4] ATLAS Collaboration, ATLAS Forward Detectors for Measurement of Elastic Scattering and Luminosity, Technical Design Report, ATLAS TDR 018, CERN/ LHCC 2008-04.

[5] S. Jakobsen, Commissioning of the Absolute Luminosity For ATLAS detector at the LHC, CERN-THESIS-2013-230 (2013).

[6] ATLAS Collaboration, Measurement of the total cross section from elastic scattering in pp collisions at $\sqrt{s}=8 \mathrm{TeV}$ with the ATLAS detector, Phys.Lett. B761 (2016) 158-178, arXiv:1607.06605 [hep-ex] CERN-EP-2016-158. 